\title{
Tuning the Photoluminescence of Graphene Quantum Dots by Fluorination
}

\author{
Yi Luo, Yongjie Xu, Ming Li, Lang Sun, Guanghui Hu, Tao Tang, \\ Jianfeng Wen, and Xinyu Li \\ College of Science, Guilin University of Technology, Guilin 541004, China \\ Correspondence should be addressed to Ming Li; liming928@163.com and Xinyu Li; lixinyu5260@163.com
}

Received 18 May 2017; Revised 13 August 2017; Accepted 14 September 2017; Published 7 November 2017

Academic Editor: Liyi Li

Copyright (c) 2017 Yi Luo et al. This is an open access article distributed under the Creative Commons Attribution License, which permits unrestricted use, distribution, and reproduction in any medium, provided the original work is properly cited.

\begin{abstract}
Fluorinated graphene quantum dots (F-GQDs) were prepared by mixing GQDs and $\mathrm{XeF}_{2}$ in a facile gaseous phase heating method. The F-GQDs with excellent water solubility have a F/C atomic ratio of $84.25 \%$ and a diameter of $2-6 \mathrm{~nm}$. The photoluminescence (PL) properties of GQDs and F-GQDs were investigated systematically. The results showed that the PL emission of the F-GQDs exhibited an obvious blue-shift of $90 \mathrm{~nm}$ compared to that of the GQDs.
\end{abstract}

\section{Introduction}

Graphene quantum dots (GQDs) have been paid attention due to their robust chemical inertness, good biocompatibility, low toxicity, and strong photoluminescence (PL) [1-5]. The modulation of the band gap of GQDs by various means has attracted great interest for potential applications. The band gap properties of GQDs are caused by quantum confinement effects [6-8]. In general, the band gap or the PL emission of GQDs can be modulated by their size and chemical functionalization. Chemical functionalization by doping foreign atoms, such as nitrogen [9], chlorine [10], and sulfur [11], is the main method for tuning the band gap of GQDs. Fluorine has been used to tune the electronic and optical properties of graphene due to its high electronegativity [12-16]. Theoretical calculations also demonstrated that the band gap of graphene can be changed from 0 to $3.13 \mathrm{eV}$ by increasing the fluorination degree, leading to a tunable optical property [17]. However, little work has been made to tune the band gap of GQDs by fluorination $[18,19]$. In practice, PL emission of these F-GQDs showed a relatively small shift (usually less than $30 \mathrm{~nm}$ ) compared to that of the GQDs. Accordingly, it is expected that the PL emission of GQDs can exhibit a large shift by fluorination.

Various methods have been realized in preparation of Fluorinated GQDs (F-GQDs), such as hydrothermal cutting routes [18] and microwave-assisted technique [19]. Nevertheless, these methods are unsatisfactory due to low yields. Therefore, it is important for both experimental studies and potentially valuable applications to develop a high-yield method for preparation of F-GQDs.

Herein, we synthesize the high-yield F-GQDs by mixing GQDs and $\mathrm{XeF}_{2}$ through a gaseous phase heating route. The obtained F-GQDs with excellent water solubility have a F/C atomic ratio of $84.25 \%$ and diameter of $2-6 \mathrm{~nm}$. The PL properties of the GQDs and F-GQDs were investigated. The PL emission of the F-GQDs exhibited a clear blue-shift of $90 \mathrm{~nm}$ compared to that of the GQDs.

\section{Methods}

GQDs were prepared in large scale through a bottom-up method as reported elsewhere [20]. F-GQDs were synthesized by mixing GQDs and $\mathrm{XeF}_{2}$ via a gaseous phase heating route. Fluorine atoms of $\mathrm{XeF}_{2}$ replaced the hydroxyl group on the surface of GQDs during the gaseous phase heating reaction. A mixture of prepared GQDs $(50 \mathrm{mg})$ and $\mathrm{XeF}_{2}$ $(250 \mathrm{mg}$ ) was first mixed carefully and transferred to a Teflon-lined stainless autoclave under an Ar atmosphere. The mixture was then heated to $180^{\circ} \mathrm{C}$, which was maintained for $12 \mathrm{~h}$. After cooling to room temperature, the obtained F-GQDs were dried at $70^{\circ} \mathrm{C}$ for $12 \mathrm{~h}$ under a vacuum to eliminate excess $\mathrm{XeF}_{2}$. 


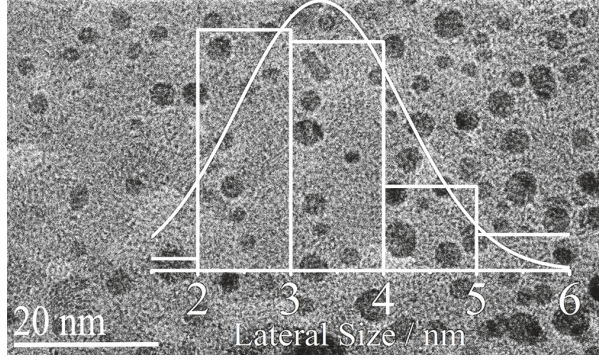

(a)

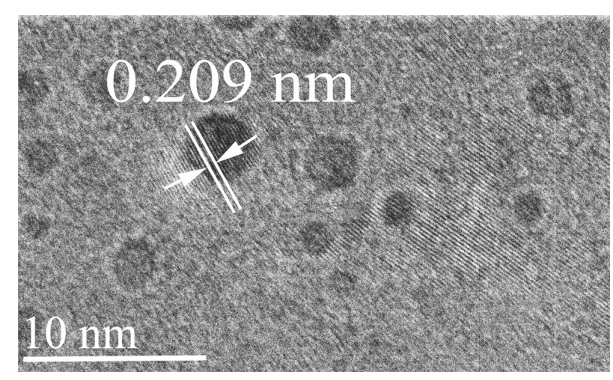

(b)

FIGURE 1: (a) TEM image (inset: diameter distribution) of F-GQDs. (b) HRTEM image of F-GQDs.

\section{Characterization}

The morphologies of the F-GQDs were characterized by transmission electron microscopy (TEM), and X-ray photoelectron spectroscopy (XPS) measurements were performed on ESCALAB using an AI Ka radiation. Ultraviolet-visible spectroscopy was characterized with an UV/VIS/NIR spectrometer. The PL spectra and fluorescence emission spectra were recorded using a fluorescence spectrophotometer. All measurements were performed at room temperature.

\section{Results and Discussion}

Figure 1(a) shows the TEM images of the synthesized F-GQDs. The F-GQDs clearly exhibit a distribution of 2-6 nm (inset of Figure 1(a)) and are obviously smaller than GQDs prepared with a distribution of 2-10 nm (Figure S1, in Supplementary Material, available online at https://doi.org/10.1155/2017/9682846), similar to a previous report [17]. This may result from further decomposition during the gaseous phase heating treatment. The high-resolution TEM (HRTEM) images (Figures 1(b) and S2) indicate the high crystallinity of both GQDs and F-GQDs with a lattice of $0.209 \mathrm{~nm}$, which is consistent with the (102) diffraction plane of $\mathrm{sp}^{2}$ graphitic carbon $[21,22]$.

The XPS measurements affirm the composition of GQDs and F-GQDs. As shown in Figure 2(a), there are peaks at $284 \mathrm{eV}$ (C 1s), $400 \mathrm{eV}$ (N 1s), and $533 \mathrm{eV}$ (O 1s), which are present in both GQDs and F-GQDs, as well as a weak signal from Na impurity. Notably, a peak at $686 \mathrm{eV}$ (F 1s) can only be observed in the XPS spectra of F-GQDs. It is obvious that the $\mathrm{F}$ atom from $\mathrm{XeF}_{2}$ has been doped into the GQDs. The atomic analyses resulting from XPS measurements are exhibited in Table S1. The unavoidable emergence of $\mathrm{O}$ and $\mathrm{N}$ in GQDs comes from the nitration process of pyrene. The C, F, and O contents of F-GQDs are $41.26 \%, 34.76 \%$, and $20.82 \%$, respectively. The $\mathrm{F} / \mathrm{C}$ atomic ratio was calculated to be $84.25 \%$. To get information on the incorporation of $\mathrm{C}, \mathrm{N}$, and F, fine-scanned XPS spectra were performed. The $\mathrm{C} 1 \mathrm{~s}$ spectra of GQDs and F-GQDs (Figures 2(b) and 2(c)) were fine-scanned into several components.

The intense peaks at $284.6 \mathrm{eV}, 285.6 \mathrm{eV}, 286.2 \mathrm{eV}$, and $288.1 \mathrm{eV}$, which are due to the graphitic $\mathrm{C}=\mathrm{C}, \mathrm{C}-\mathrm{N}$ bonding, $\mathrm{C}-\mathrm{O}$ bonding, and $\mathrm{C}=\mathrm{O}$ bonding, respectively, can be observed in the C 1s spectrum of both GQDs and F-GQDs. By contrast, some new peaks appear in the C 1s XPS spectra of F-GQDs. This analysis also demonstrates the presence of oxygen-rich functional groups that provide F-GQDs with excellent water solubility for practical applications. Combined with the clear fluorine peak at $686 \mathrm{eV}$, the intense peak at $287.1 \mathrm{eV}$ confirms the existence of $\mathrm{C}-\mathrm{F}$ covalent bonding in F-GQDs [23]. Figure 2(d) reveals two kinds of bonding in the $\mathrm{F}$ 1s spectra, $685.9 \mathrm{eV}$ owing to the semi-ionic $\mathrm{C}-\mathrm{F}$ bonding and $688.6 \mathrm{eV}$ is due to a covalent $\mathrm{C}-\mathrm{F}$ bonding pattern [24]. Obviously, $\mathrm{F}$ is in the form of functional groups within the F-GQDs, not in the form of elemental crystal physically adsorbed or wrapped within them.

The UV-vis absorption spectra of the GQDs and F-GQDs can be seen in Figure 3(a). Aqueous solutions of GQDs and F-GQDs reveal black and achromatic colors, respectively, under sunlight [25]; Figure 3(a) (inset) shows that GQDs and F-GQDs emit bright yellow and green photoluminescence under irradiation by a $365 \mathrm{~nm}$ UV lamp, respectively. The GQDs have a weak absorption at $342 \mathrm{~nm}$, which is close to previous reports [26], and a strong broad absorption at $480 \mathrm{~nm}$, which is in the visible light range. F-GQDs have a typical absorption peak at $337 \mathrm{~nm}$ corresponding to the $n \rightarrow$ $\pi^{*}$ transition of the $\mathrm{C}=\mathrm{O}$ bond. The excitation wavelength dependence of the emission wavelength and intensity is normal in carbon-based fluorescent materials. This behavior is caused by the distribution of particles of different emissive sites on each nanoparticle.

Notably, the difference of UV-vis spectra between GQDs and F-GQDs confirms that fluorination causes an obvious absorption shift toward short wavelengths. To explore the optical properties of the F-GQDs, a detailed PL study was carried out by using different excitation wavelengths. As shown in Figure 3(b), it is found that (i) the PL peak shifts to longer wavelength as the excitation wavelength is increased from 310 to $390 \mathrm{~nm}$ and (ii) on excitation at $324 \mathrm{~nm}$, the maximum PL intensity of F-GQDs appears at $450 \mathrm{~nm}$ with a Stokes shift of $126 \mathrm{~nm}(1.07 \mathrm{eV})$. Interestingly, one can find that, compared to GQDs, the maximum PL peak of the F-GQDs shows a blue-shift of $90 \mathrm{~nm}$ in the PL emission spectrum (Figure 3(c)). This indicates that the PL blue-shift may result from the relatively strong electron affinity of $\mathrm{F}$ atoms in the F-GQDs. Furthermore, the PL excitation (PLE) 


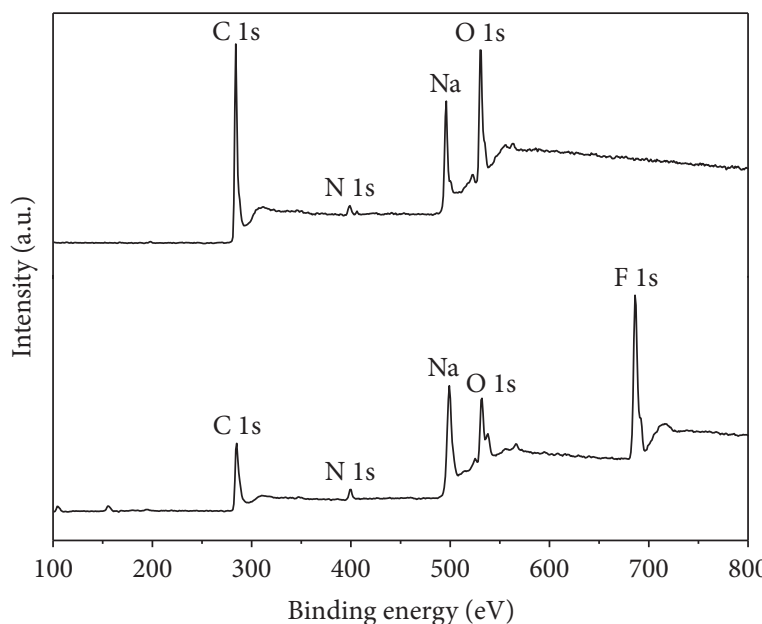

(a)

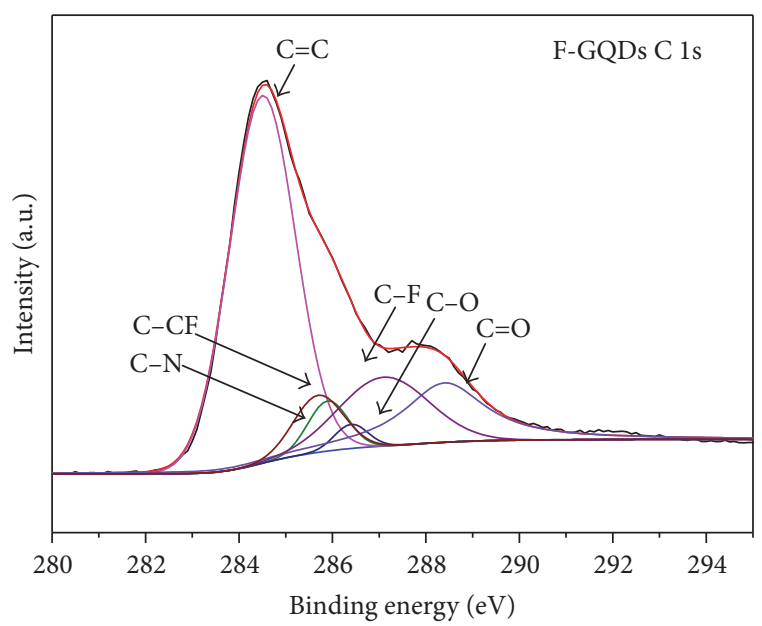

(c)

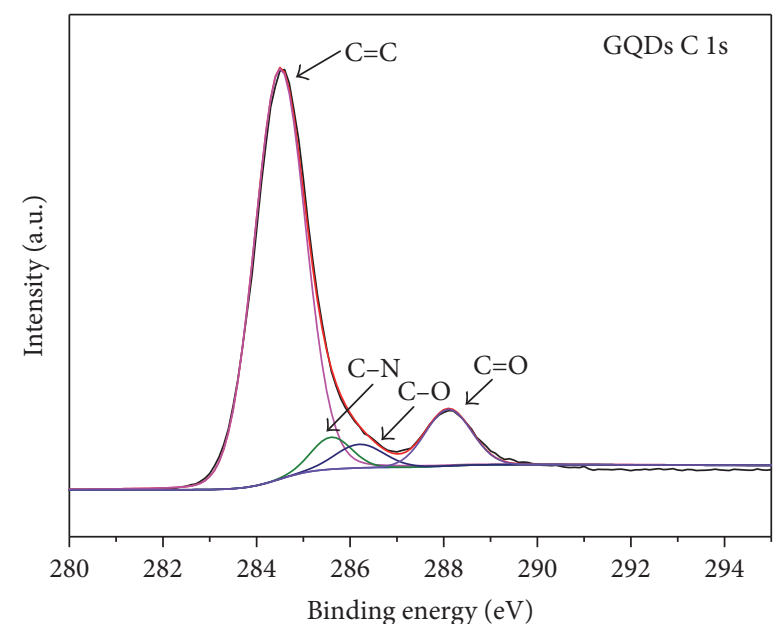

(b)

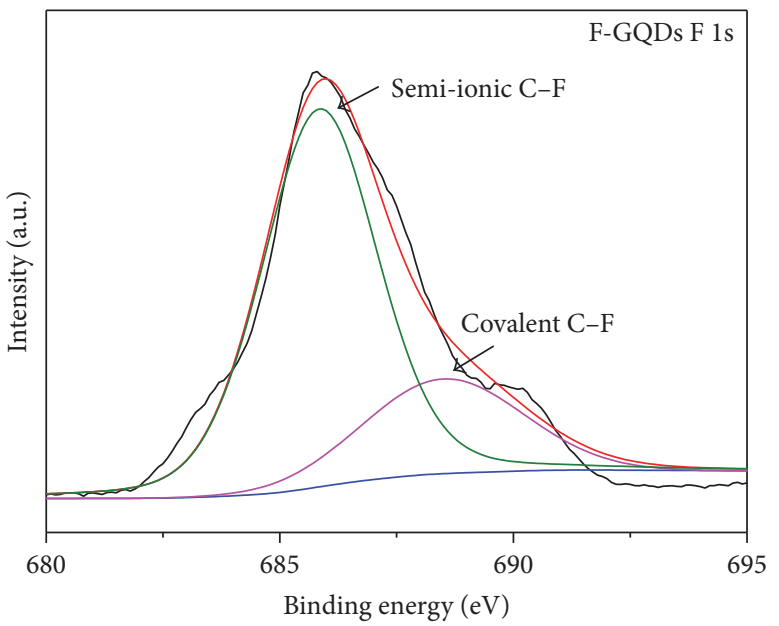

(d)

FIGURE 2: (a) XPS full spectra of GQDs and F-GQDs. High-resolution C 1s spectra of (b) GQDs and (c) F-GQDs. (d) High-resolution F 1s XPS spectrum of F-GQDs.

spectrum recorded with the strongest luminescence shows two sharp peaks at $247 \mathrm{~nm}$ and $324 \mathrm{~nm}$ (Figure 3(d)). It indicates that the luminescence of the F-GQDs is correlated with the two transitions of $247 \mathrm{~nm}(5.03 \mathrm{eV})$ and $324 \mathrm{~nm}$ $(3.83 \mathrm{eV})$, which can be regarded as transitions from the $\sigma$ and $\pi$ orbitals of $\mathrm{sp}^{2}$ triple carbenes (HOMO) to LUMO (Figure 3(e)). In addition, the quantum yield of F-GQDs is $5.2 \%$, using quinine sulfate as a reference (shown in Table S2).

To explain the above phenomena, an energy level structure model was established to study the PL properties of the F-GQDs. A schematic illustration of the typical electronic transition processes of F-GQDs is shown in Figure 3(f). The $\mathrm{PL}$ spectrum can be regarded as a transition from LUMO to HOMO. That is, when the electrons of the $\sigma$ orbital are excited, the $\sigma$ electrons transition to the LUMO, and then the electrons go back to a low-energy state [27]. As is known, this is caused by the carbene ground-state multiplicity to the energy levels of $\sigma$ and $\pi$ orbitals, which is related to the energy difference $(\delta E)$ between the $\sigma$ and $\pi$ orbitals [28]. The $\delta E$ should be below $1.5 \mathrm{eV}$, as proposed by Hoffmann [29]. Notably, the $\delta E$ in the PL process of F-GQDs is $1.2 \mathrm{eV}$. Theoretical calculations indicate that the band gap widens with increasing $\mathrm{F}$ coverage, which is due to interaction of the $\mathrm{p}$ orbitals of $\mathrm{F}$ with the $\pi$ orbitals of $\mathrm{C}$ producing $\mathrm{sp}^{3}$ bonds that modify the charge densities. As a consequence, the FGQDs show a blue-shift in the position of the PL peak with respect to that of GQDs.

In summary, we synthesized high-yield F-GQDs by mixing GQDs and $\mathrm{XeF}_{2}$ through a facile gaseous phase heating approach. The F-GQDs have a $\mathrm{F} / \mathrm{C}$ atomic ratio of $84.25 \%$ and a diameter of $2-6 \mathrm{~nm}$. The PL emission of the F-GQDs exhibits an obvious blue-shift of $90 \mathrm{~nm}$ compared to that of the GQDs. This suggests that the adsorption of fluorine on the GQDs is a promising approach to modify the properties 

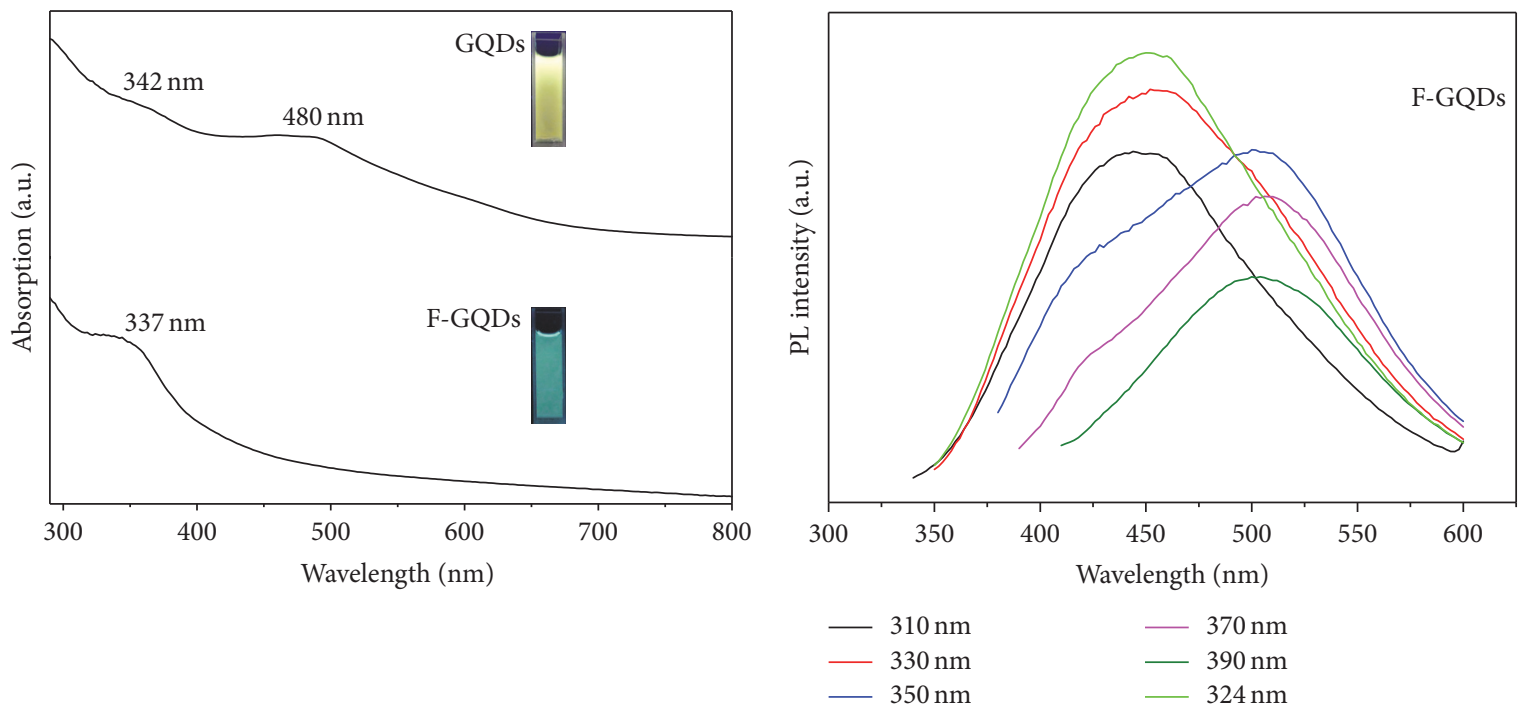

(a)

(b)
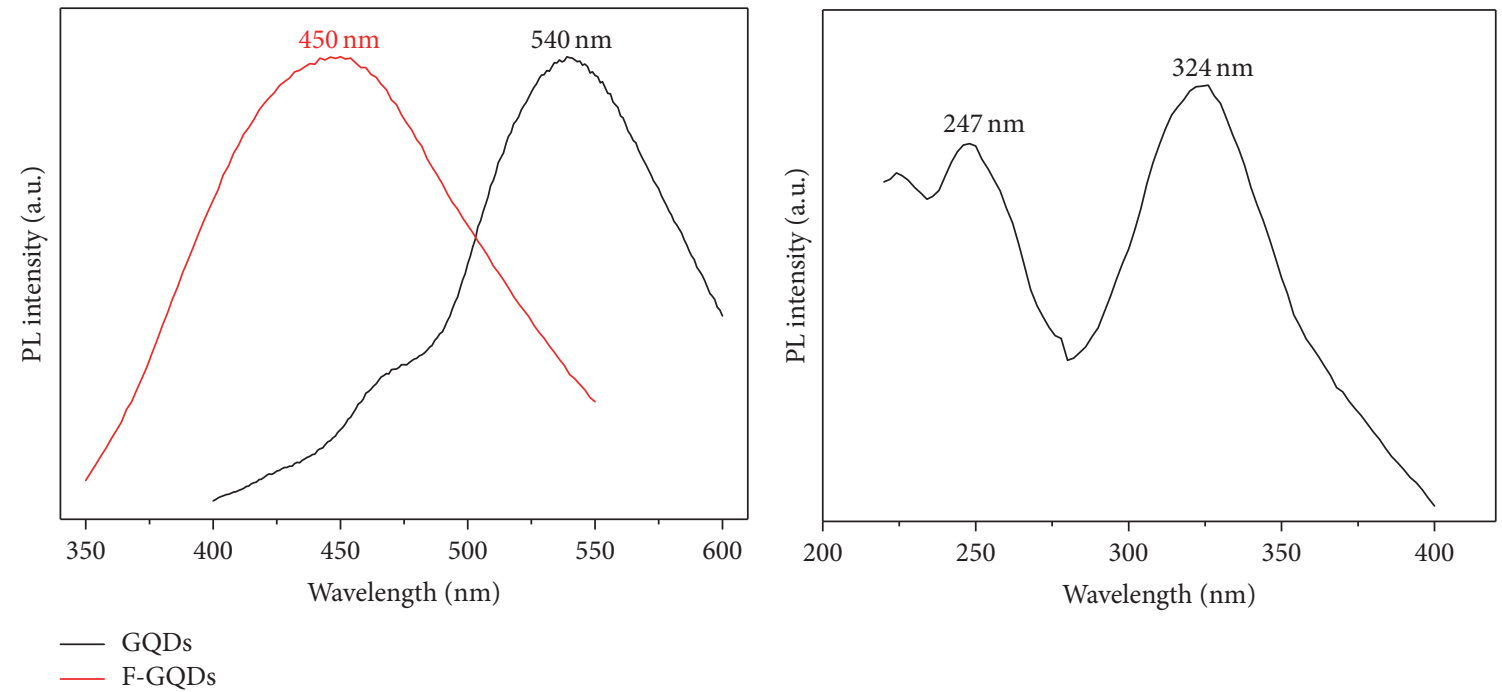

(c)

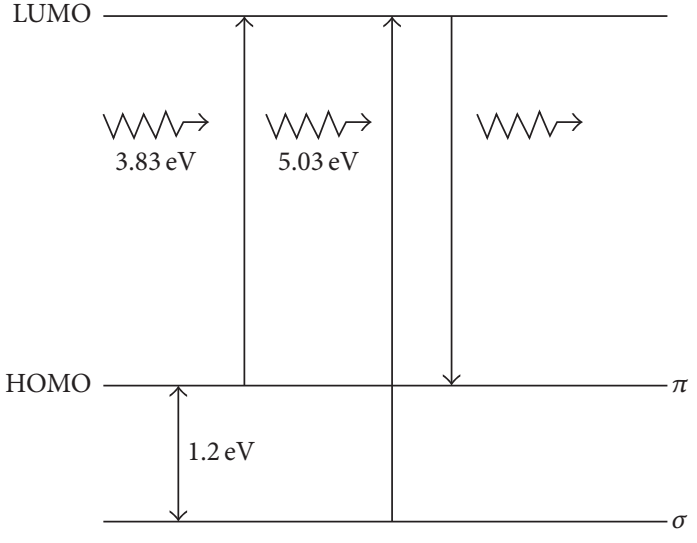

(e)

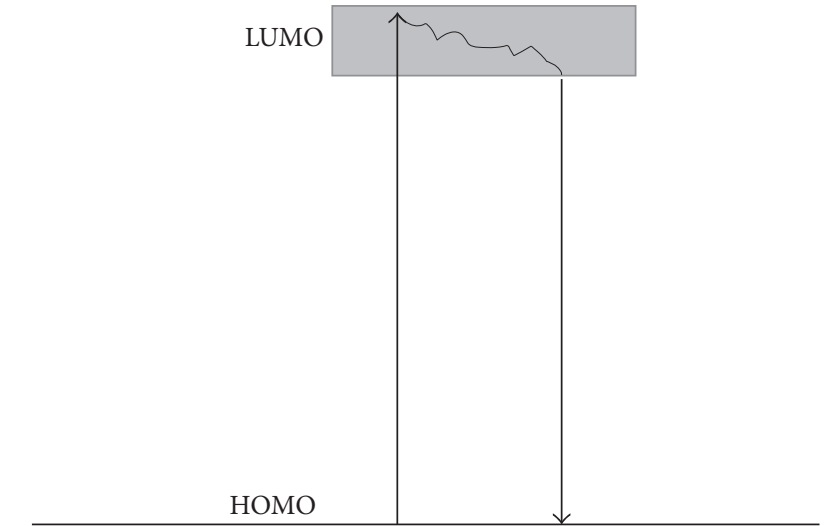

(f)

FIgURE 3: (a) The UV-vis absorption spectra of the GQDs and F-GQDs dispersed in water, and the insets are the optical photographs of the corresponding samples under the excitation with the wavelength of $360 \mathrm{~nm}$. (b) The PL spectra of F-GQDs under excitation with different wavelengths. (c) The comparison of PL spectra of GQDs with F-GQDs under the irradiation with the wavelength of $324 \mathrm{~nm}$. (d) The PLE spectrum of F-GQDs when fixing the emission wavelength at $450 \mathrm{~nm}$. (e) The typical electronic transitions of triple carbenes in the optical spectrum of the F-GQDs. (f) The schematic illustration of the typical electronic transitions processes of F-GQDs. 
of GQDs, which would lead to more flexible electrooptical applications of graphene-based materials in the future.

\section{Conflicts of Interest}

The authors declare that they have no conflicts of interest.

\section{Authors' Contributions}

Yi Luo and Yongjie Xu contributed equally to this work.

\section{Acknowledgments}

This work was financially supported by NSFC (nos. 11364010, 11764011, and 51662004) and Natural Science Foundation of Guangxi Province, China (nos. 2016 GXNSFAA380008 and 2015GXNSFBA139002).

\section{References}

[1] P. Avouris, B. Bhushan, D. Bimberg, K. v. Klitzing, H. Sakaki, and R. Wiesendanger, "Graphene quantum dots," NanoScience and Technology, 2014.

[2] P. Hawrylak, F. Peeters, and K. Ensslin, "Carbononics - integrating electronics, photonics and spintronics with graphene quantum dots," Physica Status Solidi - Rapid Research Letters, vol. 10, no. 1, pp. 11-12, 2016.

[3] R. Liu, D. Wu, X. Feng, and K. Müllen, "Bottom-up fabrication of photoluminescent graphene quantum dots with uniform morphology," Journal of the American Chemical Society, vol. 133, no. 39, pp. 15221-15223, 2011.

[4] S. Kim, S. W. Hwang, M.-K. Kim et al., "Anomalous behaviors of visible luminescence from graphene quantum dots: Interplay between size and shape," ACS Nano, vol. 6, no. 9, pp. 8203-8208, 2012.

[5] M. L. Mueller, X. Yan, J. A. McGuire, and L.-S. Li, “Triplet states and electronic relaxation in photoexcited graphene quantum dots," Nano Letters, vol. 10, no. 7, pp. 2679-2682, 2010.

[6] X. Yan, X. Cui, B. Li, and L. Li, "Large, solution-processable graphene quantum dots as light absorbers for photovoltaics," Nano Letters, vol. 10, no. 5, pp. 1869-1873, 2010.

[7] S. Neubeck, L. A. Ponomarenko, F. Freitag et al., "From one electron to one hole: Quasiparticle counting in graphene quantum dots determined by electrochemical and plasma etching," Small, vol. 6, no. 14, pp. 1469-1473, 2010.

[8] L. S. Li and X. Yan, "Colloidal graphene quantum dots," The Journal of Physical Chemistry Letters, vol. 1, no. 17, pp. 25722576, 2010.

[9] Y. Li, Y. Zhao, H. Cheng et al., "Nitrogen-doped graphene quantum dots with oxygen-rich functional groups," Journal of the American Chemical Society, vol. 134, no. 1, pp. 15-18, 2012.

[10] X. Li, S. P. Lau, L. Tang, R. Ji, and P. Yang, "Multicolour light emission from chlorine-doped graphene quantum dots," Journal of Materials Chemistry C, vol. 1, no. 44, pp. 7308-7313, 2013.

[11] D. Qu, M. Zheng, P. Du et al., "Highly luminescent S, N codoped graphene quantum dots with broad visible absorption bands for visible light photocatalysts," Nanoscale, vol. 5, no. 24, pp. 12272-12277, 2013.
[12] F. Karlický, K. Kumara Ramanatha Datta, M. Otyepka, and R. Zbořil, "Halogenated graphenes: Rapidly growing family of graphene derivatives," ACS Nano, vol. 7, no. 8, pp. 6434-6464, 2013.

[13] X. Hong, K. Zou, B. Wang, S.-H. Cheng, and J. Zhu, "Evidence for spin-flip scattering and local moments in dilute fluorinated graphene," Physical Review Letters, vol. 108, no. 22, Article ID 226602, 2012.

[14] K.-J. Jeon, Z. Lee, E. Pollak et al., "Fluorographene: A wide bandgap semiconductor with ultraviolet luminescence," ACS Nano, vol. 5, no. 2, pp. 1042-1046, 2011.

[15] J. O. Sofo, A. M. Suarez, G. Usaj, P. S. Cornaglia, A. D. Hernández-Nieves, and C. A. Balseiro, "Electrical control of the chemical bonding of fluorine on graphene," Physical Review B: Condensed Matter and Materials Physics, vol. 83, no. 8, Article ID 081411, 2011.

[16] S.-H. Cheng, K. Zou, F. Okino et al., "Reversible fluorination of graphene: Evidence of a two-dimensional wide bandgap semiconductor," Physical Review B: Condensed Matter and Materials Physics, vol. 81, no. 20, Article ID 205435, 2010.

[17] H. Y. Liu, Z. F. Hou, C. H. Hu, Y. Yang, and Z. Z. Zhu, "Electronic and magnetic properties of fluorinated graphene with different coverage of fluorine," The Journal of Physical Chemistry C, vol. 116, no. 34, pp. 18193-18201, 2012.

[18] Q. Feng, Q. Cao, M. Li, F. Liu, N. Tang, and Y. Du, "Synthesis and photoluminescence of fluorinated graphene quantum dots," Applied Physics Letters, vol. 102, no. 1, p. 013111, 2013.

[19] H. Sun, H. Ji, E. Ju, Y. Guan, J. Ren, and X. Qu, "Synthesis of fluorinated and nonfluorinated graphene quantum dots through a new top-down strategy for long-time cellular imaging," Chemistry: A European Journal, vol. 21, no. 9, pp. 3791-3797, 2015.

[20] L. Wang, Y. Wang, T. Xu et al., "Gram-scale synthesis of single-crystalline graphene quantum dots with superior optical properties," Nature Communications, vol. 5, article no. 5357, 2014.

[21] Y. Wang, L. Zhang, R.-P. Liang, J.-M. Bai, and J.-D. Qiu, "Using graphene quantum dots as photoluminescent probes for protein kinase sensing," Analytical Chemistry, vol. 85, no. 19, pp. 91489155, 2013.

[22] H. Wang, J. Xiao, Z. Yang et al., "Rational design of nitrogen and sulfur co-doped carbon dots for efficient photoelectrical conversion applications," Journal of Materials Chemistry A, vol. 3, no. 21, pp. 11287-11293, 2015.

[23] J. T. Robinson, J. S. Burgess, C. E. Junkermeier et al., "Properties of fluorinated graphene films," Nano Letters, vol. 10, no. 8, pp. 3001-3005, 2010.

[24] S. Kundu, R. M. Yadav, T. N. Narayanan et al., "Synthesis of N, F and S co-doped graphene quantum dots," Nanoscale, vol. 7, no. 27, pp. 11515-11519, 2015.

[25] Y. Dong, C. Chen, X. Zheng et al., "One-step and high yield simultaneous preparation of single- and multi-layer graphene quantum dots from CX-72 carbon black," Journal of Materials Chemistry, vol. 22, no. 18, pp. 8764-8766, 2012.

[26] D. Qu, M. Zheng, L. Zhang et al., "Formation mechanism and optimization of highly luminescent $\mathrm{N}$-doped graphene quantum dots," Scientific Reports, vol. 4, article no. 5294, no. 1, 2014.

[27] M. Li, W. Wu, W. Ren et al., "Synthesis and upconversion luminescence of N-doped graphene quantum dots," Applied Physics Letters, vol. 101, no. 10, p. 103107, 2012. 
[28] D. Pan, J. Zhang, Z. Li, and M. Wu, "Hydrothermal route for cutting graphene sheets into blue-luminescent graphene quantum dots," Advanced Materials, vol. 22, no. 6, pp. 734-738, 2010.

[29] R. Hoffmann, "Trimethylene and the addition of methylene to ethylene," Journal of the American Chemical Society, vol. 90, no. 6, pp. 1475-1485, 1968. 

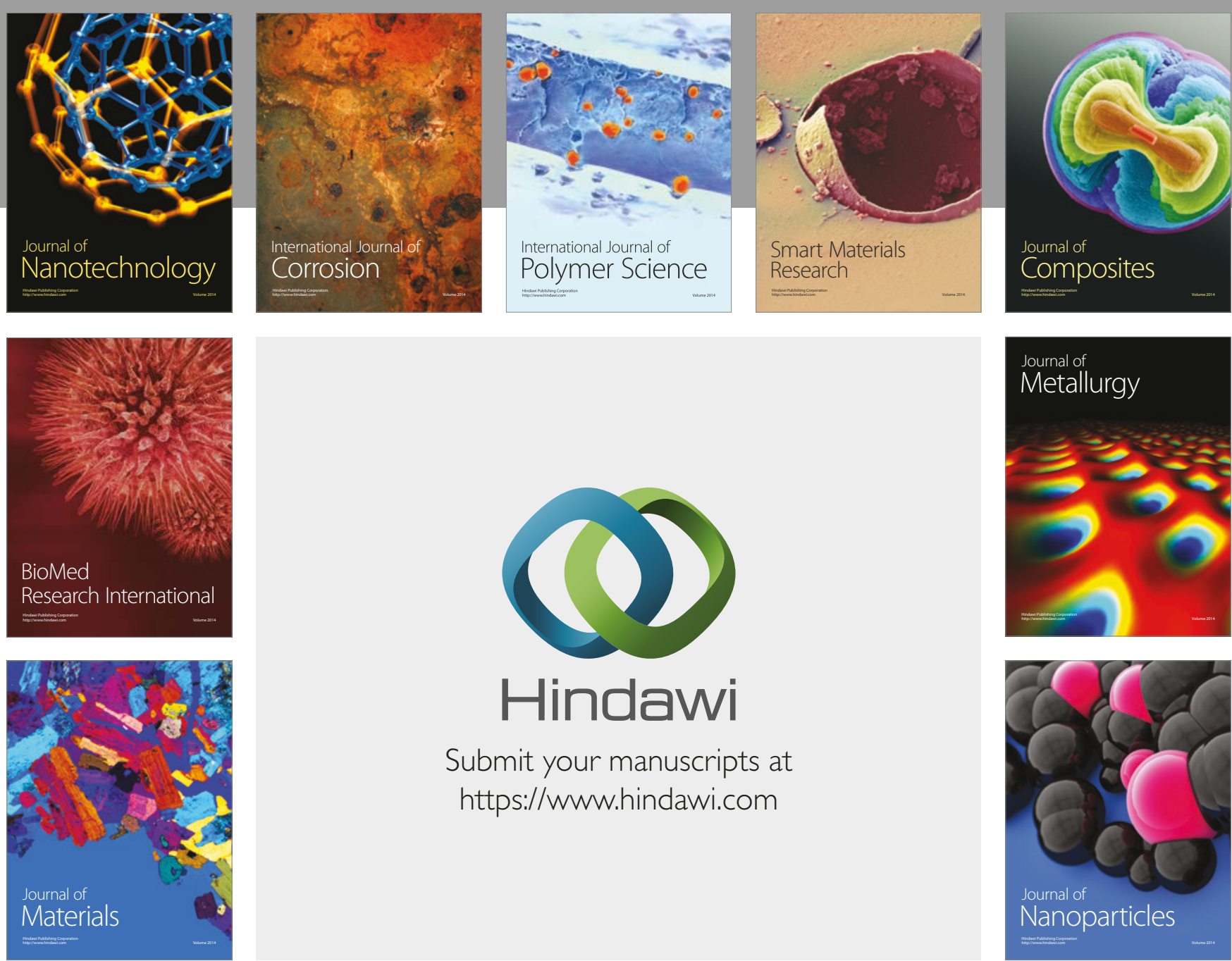

\section{Hindawi}

Submit your manuscripts at

https://www.hindawi.com
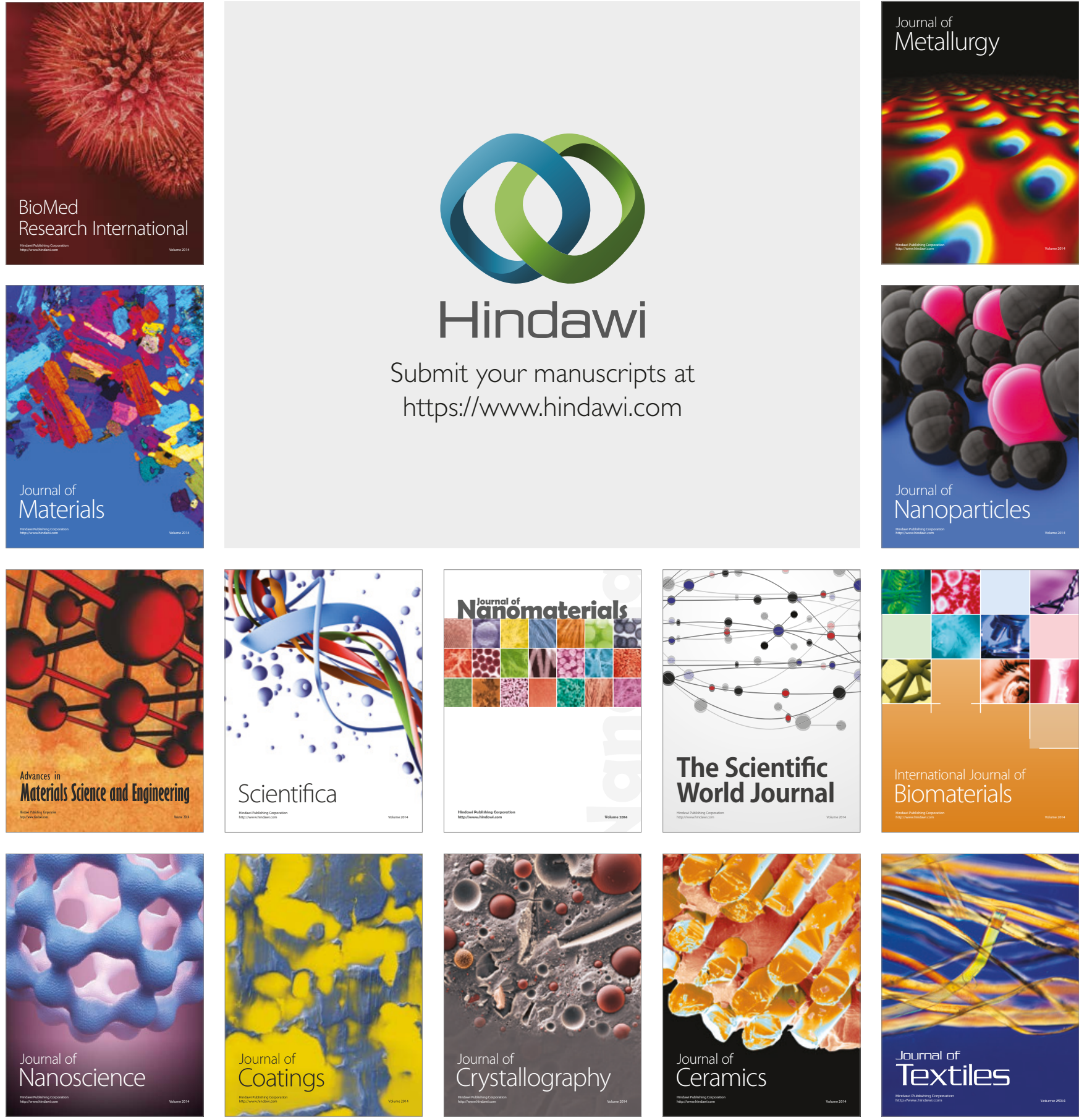

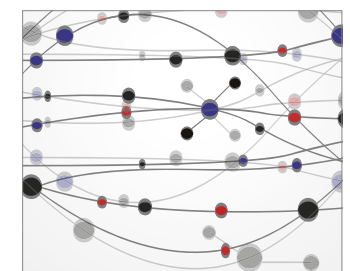

The Scientific World Journal
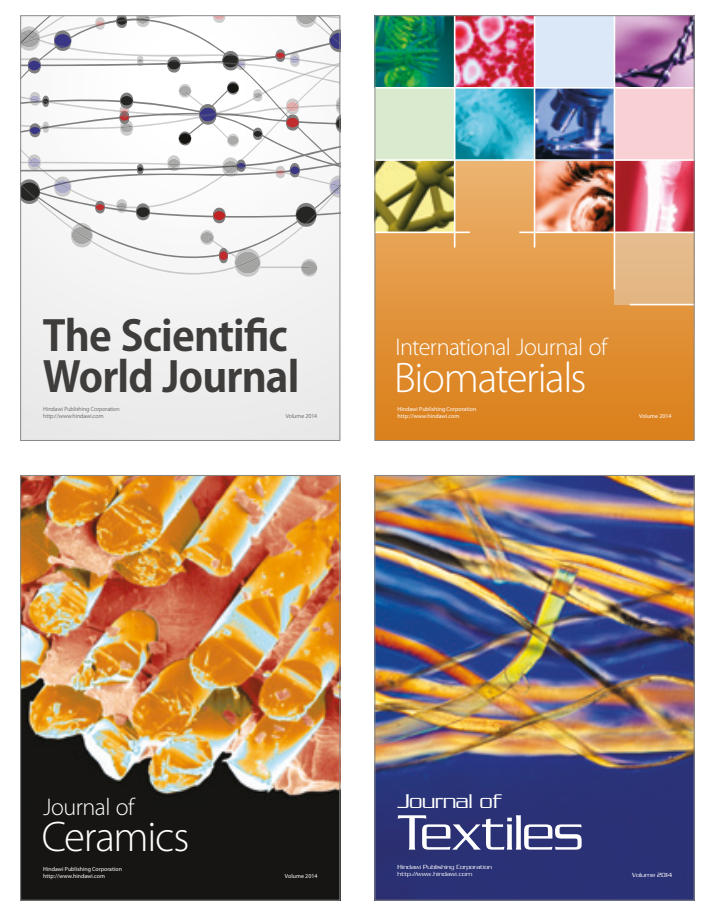